P0114

\title{
LED FILAMENT STANDARD LAMPS FOR TOTAL LUMINOUS FLUX AND LUMINOUS INTENSITY
}

\author{
J. Y. YAN et al.
}

DOI 10.25039/x46.2019.PO114

from

CIE x046:2019

Proceedings

of the

29th CIE SESSION

Washington D.C., USA, June 14 - 22, 2019

(DOI 10.25039/x46.2019)

The paper has been presented at the 29th CIE Session, Washington D.C., USA, June 14-22, 2019. It has not been peer-reviewed by CIE.

(c) CIE 2019

All rights reserved. Unless otherwise specified, no part of this publication may be reproduced or utilized in any form or by any means, electronic or mechanical, including photocopying and microfilm, without permission in writing from CIE Central Bureau at the address below. Any mention of organizations or products does not imply endorsement by the CIE.

This paper is made available open access for individual use. However, in all other cases all rights are reserved unless explicit permission is sought from and given by the $\mathrm{CIE}$.

CIE Central Bureau

Babenbergerstrasse 9

A-1010 Vienna

Austria

Tel.: +4317143187

e-mail: ciecb@cie.co.at

www.cie.co.at 


\title{
LED FILAMENT STANDARD LAMPS FOR TOTAL LUMINOUS FLUX AND LUMINOUS INTENSITY
}

\author{
Yan, J.Y., Liu, H., Zhao, W.Q., Su, Y., Jiang, L. \\ National Institute of Metrology, Beijing, CHINA \\ yanjy@nim.ac.cn
}

DOI 10.25039/x46.2019.PO114

\begin{abstract}
Light emitting dioxides (LEDs) would inevitably replace the incandescent lamps and dominate the lighting market. There is a growing demand for LED measurement and calibration. However, using the incandescent lamp as a standard lamp to measure LEDs would produce great uncertainty. LED filament standard lamps for total luminous flux and luminous intensity are developed to solve this problem. Both the intensity of type A and type B standard LED for luminous flux are uniformly distributed over $4 \pi$ geometry. The angular uniformity and conformity of the inverse square law of the standard LED for luminous intensity are well satisfied. The developed LED standard have a shorter warm-up time, good short-term stability and repeatability. The luminous flux and luminous intensity are increased by $1.5 \% \sim 2.5 \%$ after 2000 hours aging.
\end{abstract}

Keywords: LED filament, Standard lamp, Photometry

\section{Introduction}

The light emitting dioxide (LED) light source has the outstanding advantages of long life and high luminous efficiency. In terms of energy saving, traditional incandescent lamps and fluorescent lamps are being phased out worldwide in general illumination applications. This led to great challenges in photometry. Firstly, Incandescent lamps are still the main standards in current photometric metrology systems. When using incandescent lamps as the standard lamps to measure the LEDs will introduce great uncertainty due to the significant spectrum difference between LED lights and traditional incandescent lights (Ohno et al., 2014), Secondly, it is becoming more and more difficult and expensive to supplement the incandescent standard lamps.

For these reasons, CCPR, CIE, and some national metrology institutes have paid attention to the development of LED-based photometric standards. The Consultative Committee for Photometry and Radiometry (CCPR) Working Groups (WGs) have established a Task Group: "WG-KC TG4: Pilot Study for the use of alternative standards for photometric comparisons" (BIPM, 2013). CIE lists "New Calibration Sources and Illuminants for Photometry, Colorimetry and Radiometry" as one of the top ten strategic plans. NMIJ (Japan) developed a new standard LED of total spectral radiant flux in $2 \pi$ geometry (Nakazawa et al., 2018). PTB (Germany) developed 2 types of high power $(100 \mathrm{Im}$ and $2500 \mathrm{Im})$ LED-based calibration standards for luminous flux (Zwinkels, 2016). All these LED standards installed thermomodule to keep a constant temperature. However, the thermo-controllers are cumbersome and a power supply system is needed. It is difficult to be compatible with existing photometric systems and is not convenient for use in industrial applications.

NIM (China) developed the first generation of LED filament lamp for total luminous flux in 2016, see Figure 1. This lamp exhibits a short warm-up time $(<10$ minutes $)$ and very good repeatability and reproducibility. The descent of total luminous flux is $2.0 \%-6.8 \%$ after continuously ignited for 9700 hours, which is $1 / 30$ of that of the incandescent lamp. One of the most outstanding advantages is its good heat dissipation without requiring additional heat dissipation and cooling. The connector interface is E27 which is fully compatible with incandescent lamps and easy to popularize (Liu et al., 2017). However, the intensity of this standard lamp is weaker in the axial direction of $180^{\circ}$, as shown in Figure 1. 
In this paper, we developed the second generation of LED filament lamp for total luminous flux with a uniform intensity distribution over $4 \pi \mathrm{sr}$. Moreover, we also developed a LED filament lamp for luminous intensity.
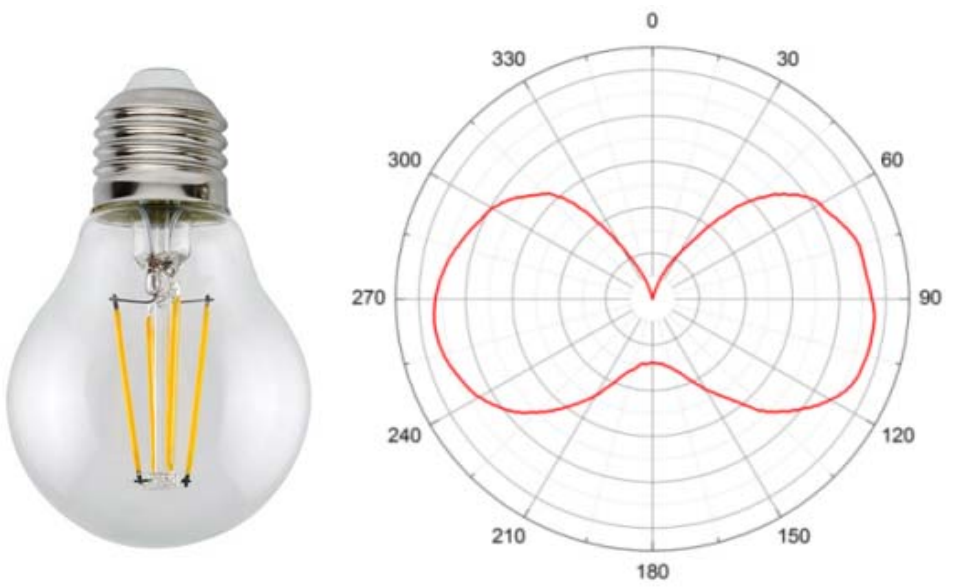

Figure 1 - NIM 1st generation of LED filament standard lamp for total luminous flux. Left: photo of the lamp; Right: luminous intensity distribution in the vertical plane.

\section{Development of the LED filament standard lamps}

Standard lamps are used to preserve and transfer photometric values. There are two important standard lamps. One is for total luminous flux and the other is for luminous intensity. At the CCPR WG-TG4 meeting, recommendations were made for both types of LED standard lamps for international comparisons. The luminous flux level is $(1000 \sim 3000) \mathrm{Im}$ and the angular intensity distribution is nearly omnidirectional. The luminous intensity level is $(200 \sim$ $500) \mathrm{cd}$ and the angular intensity distribution is close to Lambertian. The relative spectral radiant flux is close to $4100 \mathrm{~K}$ LED reference spectrum. Long-term stability is tested operating the lamps for 1 hour during consecutive 210 days and measurement every 21 days. Short term stability and ambient temperature sensitivity are provided by each lab.

Among many other types of LED lamps, such as an LED bead, LED spot lamp, LED tube, etc. LED filament lamp is very suitable to develop for both standard lamps and easy to substitute for the traditional incandescent standard lamps.

\subsection{General purpose design}

LED is a kind of semiconductor device. Its luminous efficiency is sensitive to the LED die temperature. The variation of the temperature gradient between the LED die and the ambient lead to re-establish thermal equilibrium. The typical temperature coefficient of LEDs is about $(0.15 \sim 0.30) \% /{ }^{\circ} \mathrm{C}$. A good thermal design can keep the LED die temperature in a lower range and shorten the time required for thermal equilibrium.

In the design of our LED standard, the LED filament is used. A LED filament is composed of 28 or more LED dies which are fixed in series on the sapphire substrate. Four or more LED filaments with certain arrangement compose a lamp stem which is enclosed with a G125 or G150 glass bulb. The bulb is filled with inert gas, and the heat of the filament is quickly transferred to the bulb surface by the inert gas. Thus, the thermal equilibrium is soon achieved and the junction temperature of the LED chip can be controlled to a lower range. Meanwhile, the shape and the connector interface are fully compatible with incandescent lamps. Unlike the flexible tungsten filament, the LED filament is rigid. So that the LED filament standard lamp is shock resistant.

The current photometric system is based on illuminant $A$ with a relative spectral power distribution that is consistent with a blackbody radiation of $2856 \mathrm{~K}$. As LEDs gradually replaced incandescent lamps, the standard illuminant $L$, which represents the typical spectrum of LEDs, was incorporated into the CIE standard illuminant with a correlated colour 
temperature (CCT) of $4102 \mathrm{~K}$ and a spectrum as shown in Figure 2. The spectral bandwidth of a single LED die is narrow. To achieve a wide spectral bandwidth, there are usually two ways: one is to use multiple LED chips with different peak wavelengths and packaged together according to a specific arrangement. Another way is to coat the blue LED die with yellow phosphor (Nakazawa et al., 2018). The developed LED filament standard lamp adopts the latter method, and its spectrum and CCT are close to the CIE illuminant L, as shown in Figure 2.

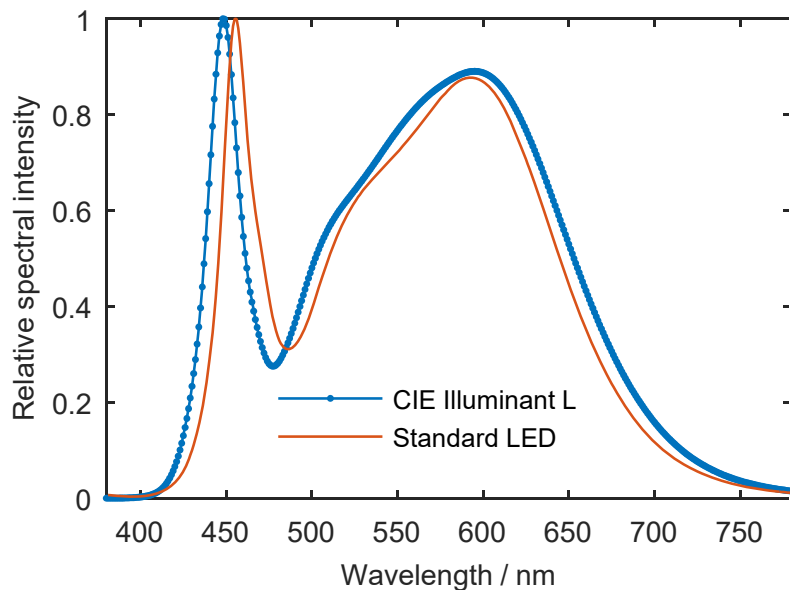

Figure 2 - Typical spectrums of CIE illuminant L and LED filament standard.

\subsection{LED filament standard lamp for total luminous flux}

The standard lamp for total luminous flux is used to preserve and calibrate the total luminous flux value. To measure the total luminous flux of a lamp, a goniophotometer and an integrating sphere are used for absolute or comparative measurements. When using a goniophotometer, the detector samples the spatial intensity distribution of the lamp and integrates the intensity values in each direction to obtain the total luminous flux. In practice, the intensity of the sampling direction represents the average intensity near the current direction. If the spatial intensity distribution of the lamp is not uniform, the uncertainty of the total luminous flux will increase. When the total luminous flux is measured by an integrating sphere, since the integrating sphere coating reflectivity is not completely consistent, the spatial nonuniformity of the intensity distribution of the lamp would also increase the measurement uncertainty. Thus, the standard lamp for total luminous flux requires uniform intensity distribution in $4 \pi$ geometry.

Two types of standard LED filament lamp for total luminous flux are developed. As shown in Figure 3, The LED filaments are arranged symmetrically with a tilt. In this way, a uniform intensity distribution over $4 \pi$ steradian is realized. The typical parameters are: (Type A) 450 Im, DC 20 mA, $128 \mathrm{~V}$; (Type B) $800 \mathrm{Im}$, DC $32 \mathrm{~mA}, 128 \mathrm{~V}$. Both of the types are E26-based.
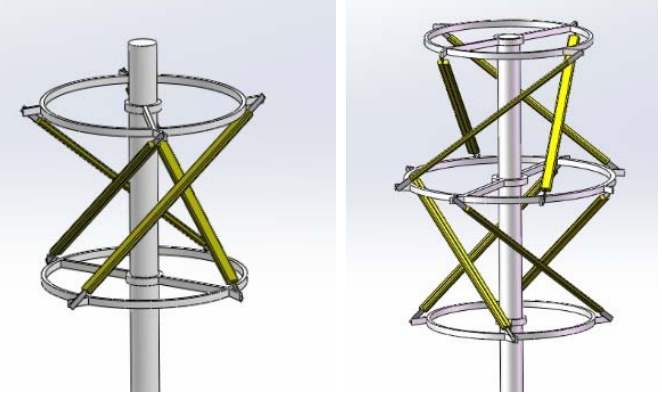

Figure 3 - The filament arrangement of LED standard for total luminous flux. Left: type A, Right: type B. 


\subsection{LED filament standard lamp for luminous intensity}

A LED standard lamp for luminous intensity is used to provide its value in the direction of the plane normal. The plane is composed of 12 LED filaments, which are parallel to each other and equally spaced. The size of the filament plane is $60 \mathrm{~mm} \times 39 \mathrm{~mm}$, as shown in Figure 4 . A G150 glass bulb is selected as the shell. To ensure the repeatability of the standard lamp, it is necessary to evaluate the angular uniformity of luminous intensity. The good angular characteristic makes the luminous intensity insensitive to the alignment. The standard lamp for luminous intensity is usually used for the calibration of the illuminance meter and the luminance meter. In order to evaluate the linearity of the meter, the illuminance is measured at different distances on the photometry bench, and is calculated as:

$$
E=\frac{I}{l^{2}}
$$

where

$$
\begin{array}{ll}
E & \text { is the illuminance; } \\
l & \text { is the distance; } \\
I & \text { is the luminance. }
\end{array}
$$

Equation (1) is well known as the inverse square law between illuminance and distance. However, due to the imperfect transmittance of the glass bulb and the luminous intensity nonuniformity, the conformity of the law should be assessed.

With these considerations, the LED filament standard lamp for luminous intensity is developed, and the photo of the lamp is shown in Figure 4. The typical parameters of are: $170 \mathrm{~cd}, \mathrm{DC} 120$ $\mathrm{mA}, 65 \mathrm{~V}$. The interface is E40-based.
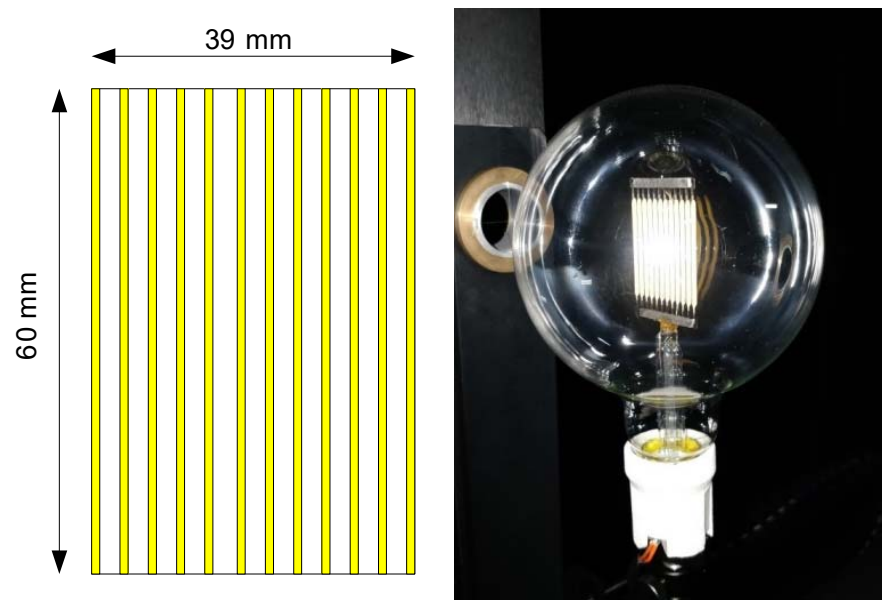

Figure 4 - LED filament standard lamp for luminous intensity. Left: the arrangement and the size of the LED filaments plane, Right: photo of the lamp.

\section{Results and discussion}

The LED filament standard lamps are carefully tested. The long-term and short-term stability are tested in the sphere photometer. The angular intensity distribution is tested on the goniophotometer. The angular uniformity of luminous intensity is measured on the optical bench. The ambient temperature dependence was evaluated using a thermostatic chamber.

\subsection{LED filament standard lamp for total luminous flux}

\subsubsection{Spatial characteristics}

The spatial characteristics of the developed standard lamps are tested using the NIM's goniophotometer. In the test, type $A$ and $B$ LED standard operated at $20 \mathrm{~mA}$ and $32 \mathrm{~mA}$ respectively. The luminous intensity distributions of the LED standard type $A$ and $B$ are shown 
in Figure 5 in a polar coordinate system. The E26-base is in the direction of $\theta=0^{\circ}$ vector and the luminous intensity is blocked. As shown in Figure 5, both luminous intensity distributions of type $A$ and $B$ standard LED are uniformly distributed in $4 \pi \mathrm{sr}$.
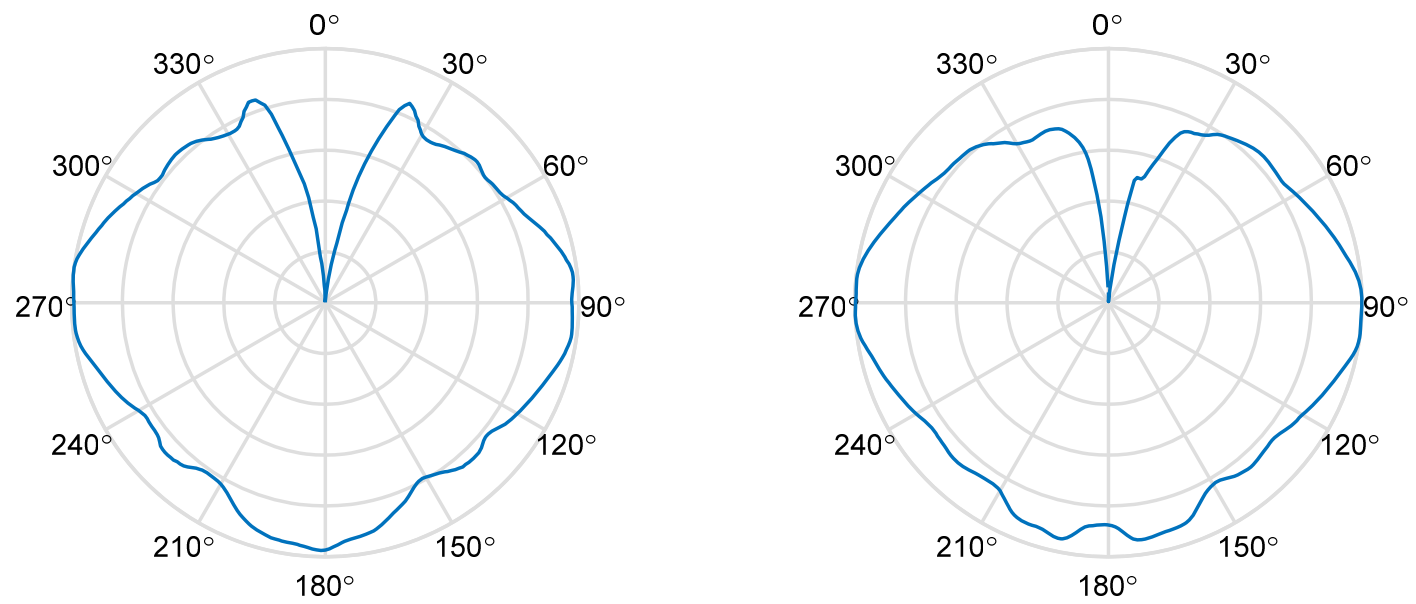

Figure 5 - The luminous intensity distribution of the LED filament standard lamp for total luminous flux. Left: type A \#63, Right: type B \#42.

\subsubsection{Stability}

The short-term stability of a LED filament standard lamp for total luminous flux was investigated at an ambient temperature of $(22.6 \pm 0.1){ }^{\circ} \mathrm{C}$. The lamp was operated for 4 hours, then it was turn off for an hour. This process was repeated for 3 times under the same conditions and the relative luminous flux was recorded and normalized, as illustrated in Figure 6.

Figure 6 demonstrates that the luminous flux stabilized quickly within 8 minutes, and the relative standard deviation of each 4 -hours-test process is less than $0.002 \%$. The short-term stability is better than $0.01 \%$, the repeatability is better than $0.02 \%$ and the warm-up time is less than $8 \mathrm{~min}$.

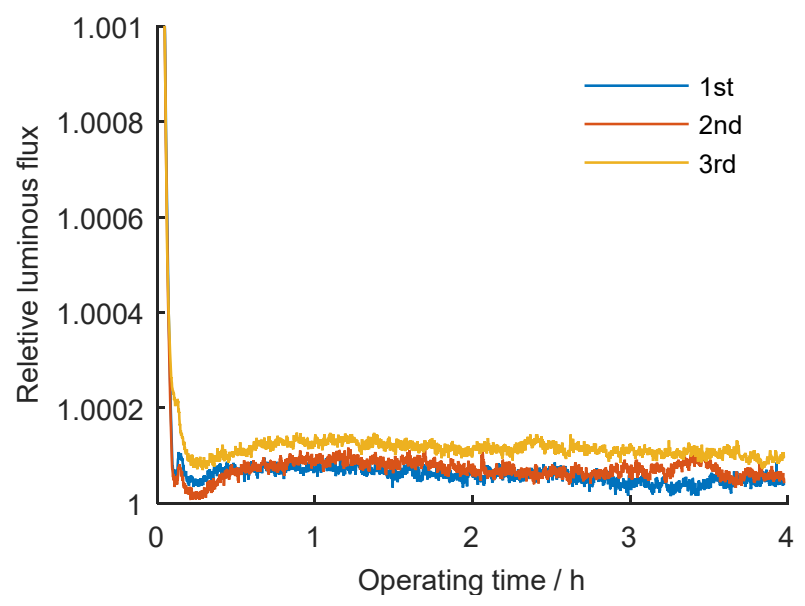

Figure 6 - Short-term stability of the standard LED for total luminous flux.

The long-term stability of LED filament standard lamps for total luminous flux is also evaluated. The three standard LEDs continuously operated for 2744 hours at their rated current $20 \mathrm{~mA}$. The luminous flux of all lamps is normalized at the first measurement. The result is shown in Figure 7 . The luminous flux is increased by less than $2.5 \%$ after 2700 hours of burning. In contrast, the luminous flux of incandescent standard lamps usually decreases by $2 \%$ after 100 hours of burning. 


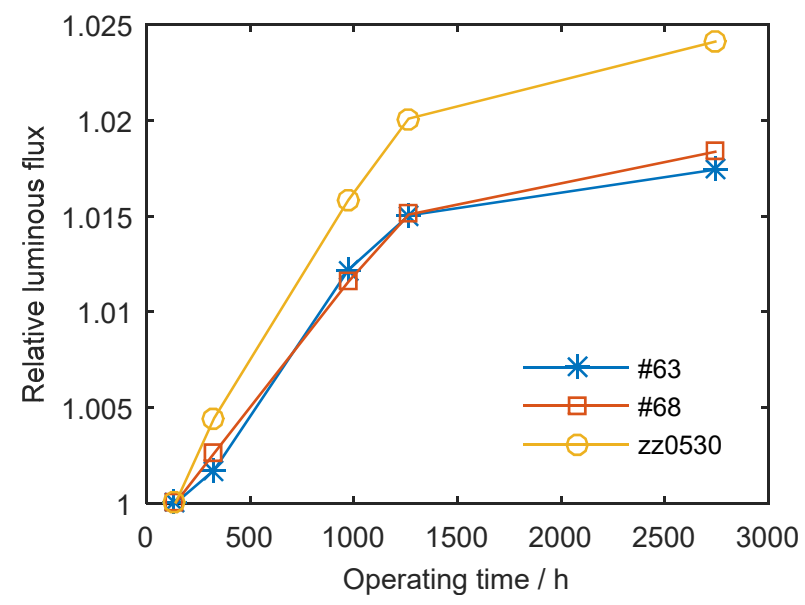

Figure 7 - Long-term stability of the standard LED for total luminous flux.

\subsubsection{Ambient temperature dependence}

The LED standard lamp was set in the chamber while the photometric detector was set out of the chamber. The radiation emitted from the test lamp was measured through a chamber window at 4 temperatures $\left(32.0^{\circ} \mathrm{C}, 37.4^{\circ} \mathrm{C}, 42.2^{\circ} \mathrm{C}, 46.8^{\circ} \mathrm{C}\right)$, see Figure 8 .

As shown in Figure 8, the temperature coefficient is $0.17 \% /{ }^{\circ} \mathrm{C}$. Most LEDs with good thermal control have a thermal sensitivity of $(0.1 \sim 0.3) \% /{ }^{\circ} \mathrm{C}$.

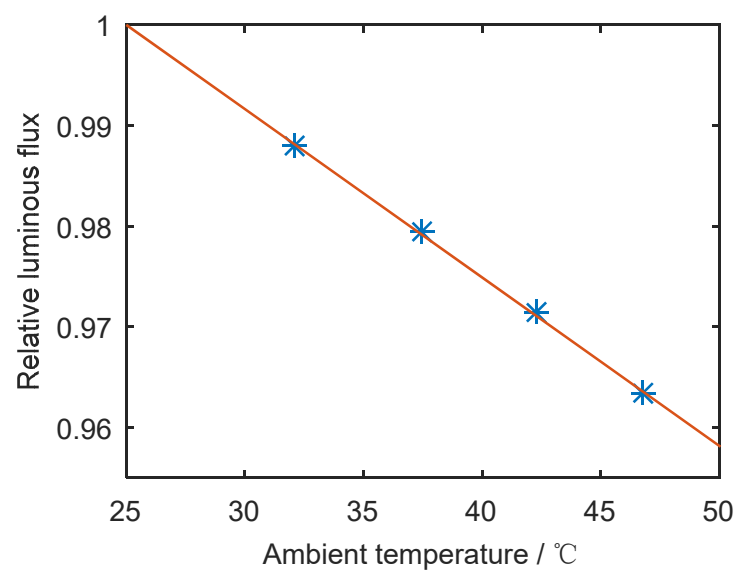

Figure 8 - Ambient temperature dependence of the standard LED for total luminous flux.

\subsection{LED filament standard lamp for luminous intensity}

\subsubsection{Angular uniformity}

When using the standard lamp for luminous intensity, it is necessary to adjust the filament plane to be perpendicular to the optical axis on the photometric bench. Good angular uniformity will ease the difficulty of the alignment and help to decrease the uncertainty. The angular uniformity of the incandescent standard lamp in verification procedure JJG 246-2005 are specified: when the filament plane is rotated horizontally by $1^{\circ}$, the intensity changes less than $0.6 \%$, and when the vertical rotation is $1.5^{\circ}$, the intensity changes less than $0.6 \%$ (JJG, 2005). In NIST measurement services (NIST, 2018), the variation of luminous intensity of an FEL quartz-halogen lamp is not to exceed $\pm 0.5 \%$ in a $\pm 1^{\circ}$ rectangular region around the optical axis.

GQ \#11 lamp was selected to evaluate the angular uniformity. The results depicted in Figure 9 show that when the vertical rotation is in the range of $\pm 1.0^{\circ}$, the intensity variation is better than $0.03 \%$, and when the horizontal intensity rotation is within $\pm 1.5^{\circ}$, the intensity variation is 
less than $0.3 \%$. The requirement of the angle characteristic of the LED filament standard lamp can be satisfied.
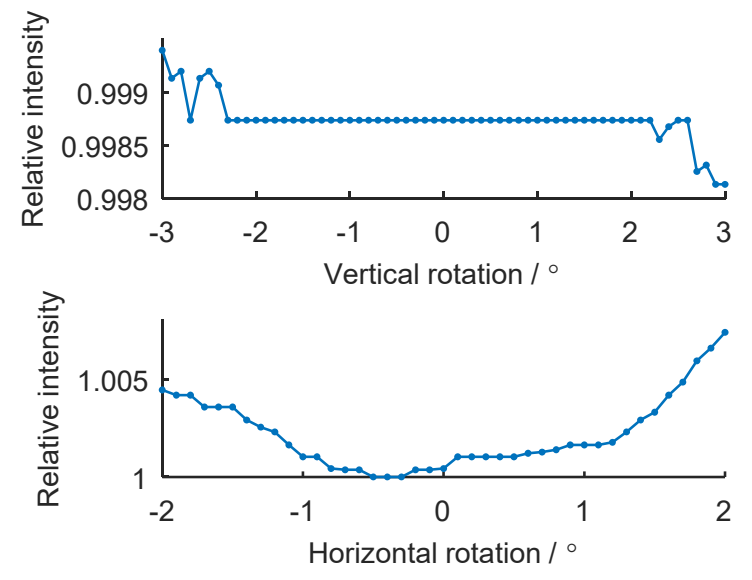

Figure 9 - Angular uniformity of GQ\#11 LED filament standard.

\subsubsection{Conformity of the inverse square law}

When the size of the source is much smaller than the distance from the detector to the source, the illuminance and distance are in accordance with inverse square law. However, due to the influence of the glass bulb, the law is not always correct. It is important to assess the conformity of the inverse square law.

The illuminance of GQ\#11 LED filament standard lamp at different distances in the range of $2.6 \mathrm{~m} \sim 11 \mathrm{~m}$ was measured. The intensity and its relative deviation from the average are shown in Table 1.

Table 1 - Luminous intensity and its relative deviation of GQ\#11 at different distances.

\begin{tabular}{|c|c|c|}
\hline Distance / mm & $\begin{array}{c}\text { Luminous } \\
\text { intensity / cd }\end{array}$ & $\begin{array}{c}\text { Relative } \\
\text { deviation }\end{array}$ \\
\hline 2663.9 & 169.39 & $-0.04 \%$ \\
\hline 3548.5 & 169.49 & $0.01 \%$ \\
\hline 4863.8 & 169.85 & $0.23 \%$ \\
\hline 6726.2 & 169.52 & $0.04 \%$ \\
\hline 7715.0 & 169.70 & $0.14 \%$ \\
\hline 8617.0 & 169.44 & $-0.01 \%$ \\
\hline 10268.5 & 169.66 & $0.12 \%$ \\
\hline 11033.9 & 169.84 & $0.22 \%$ \\
\hline Mean & $169.46 \mathrm{~cd}$ & \\
\hline
\end{tabular}

As seen from Table 1, the maximum deviation is $0.23 \%$ with respect to the average, which is equivalent to the level of incandescent lamps.

\subsubsection{Stability}

The short-term and long-term stability of a LED filament standard lamp for luminous intensity was evaluated similar with the lamp for luminous flux.

Figure 10 demonstrates that the warm-up time is about 10 minutes. The relative standard deviations of three consecutive measurements of 5.7 hours are $0.012 \%, 0.008 \%$, and $0.007 \%$, respectively. The short-term stability is better than $0.03 \%$.

The long-term stability of LED filament standard lamps for luminous intensity is shown in Figure 11. Two standard LEDs (GQ\#10 and GQ\#11) were operated for 2049 hours at their rated current $120 \mathrm{~mA}$. The luminous flux is increased by less than $1.5 \%$ after 2000 hours of burning. 


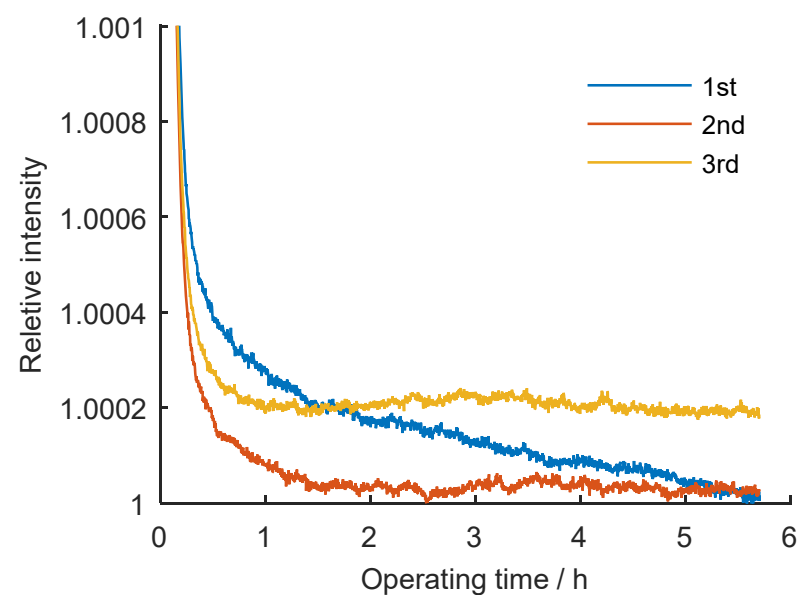

Figure 10 - Short-term stability of the standard LED for luminous intensity.

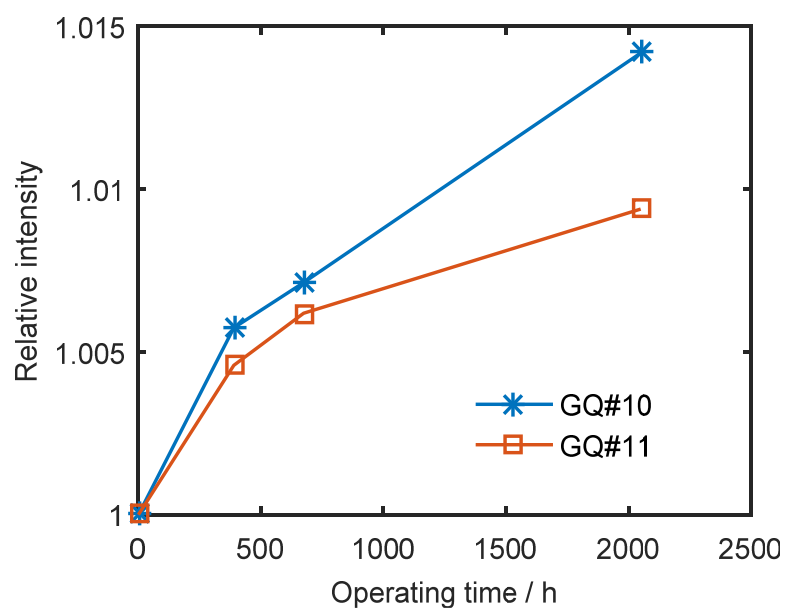

Figure 11 - Long-term stability of the standard LED for luminous intensity.

\section{Conclusions}

Two types of LED filament standard lamp of total luminous flux and a LED filament standard lamp for luminous intensity are developed. The filaments with a CCT of $4100 \mathrm{~K}$ are subtly arranged so that the standard LED for total luminous flux has a uniform distribution over $4 \pi$ steradian, the angular intensity uniformity and the conformity of the inverse square law of the standard LED for luminous intensity is comparable to the incandescent.

The heat dissipation of the filament is fast, and the warm-up time is about 10 minutes while the other LED lamp needs about 30 minutes. The short-term stability of luminous flux and intensity are better than $0.01 \%$ and $0.30 \%$, respectively. The long-term stability of luminous flux and intensity is $1.5 \% \sim 2.5 \%$. The excellent properties make the LED filament standard lamps could be good candidates for replacing incandescent standard lamps.

\section{References}

BIPM 2013: CCPR WG Strategy Document for Rolling Development. http://www.bipm.org/utils/en/pdf/CCPR-strategy-document.pdf

JJG 2005: Chinese national standard JJG 246-2005. Verification Regulation of Standard Lamp for Luminous Intensity.

LIU, H. 2017. The Development of LED Filament Standard Lamp. China Illuminating Engineering Journal, 2: 13-16. 
NAKAZAWA Y., GODO K., NIWA K., ZAMA T., YAMAJI Y., and MATSUOKA S. 2018. Development of LED-based Standard Source for Total Luminous Flux Calibration. Lighting. Res. Technol., 0: 1-13.

NIST 2018. NIST Special Publication 250-95. NIST Measurement Services Photometric Calibrations. https://doi.org/10.6028/NIST.SP.250-95

OHNO, Y., NARA, K., REYTOVA, E., ZHANG, W., ZAMA, T. and MILLER, C. 2014. Solid State Lighting Annex: 2013 Interlaboratory Comparison Final Report, online, IEA webpage http://ssl.iea4e.org/files/otherfiles/0000/0067/IC2013_Final_Report_final_10.09.2014a.pdf

ZWINKELS, J.C. 2016. CCPR Activities Related to LED-Based Calibration Standards. CIE 2016. 